RASĀYAN J. Chem.

Vol. 13 | No. 3 |1735-1743| July - September | 2020 ISSN: 0974-1496 | e-ISSN: 0976-0083 | CODEN: RJCABP

\title{
ENVIROCAT EPZG AS A HETEROGENEOUS CATALYST FOR THE SYNTHESIS OF 3,3-DISUBSTITUTED OXINDOLES
}

\author{
Rahul Patil ${ }^{1, *}$, Uday Lad ${ }^{1}$, Suresh Shendage ${ }^{2}$ and Uttam More ${ }^{3}$ \\ ${ }^{1}$ Department of Chemistry, Yashwantrao Chavan College of Science Karad, Maharashtra, India. \\ ${ }^{2}$ KET'S Vinayak Ganesh Vaze College of Arts, Science and Commerce, Mithagar Road, \\ Mulund-Mumbai 400081, India \\ ${ }^{3}$ Department of Chemistry, Sadguru Gadage Maharaj College, Karad, Maharashtra, India. \\ E-mail: rspatilorg@gmail.com
}

ABSTRACT

Synthesis of 3,3-Disubstituted Oxindoles was achieved by one-pot multicomponent condensation of isatin, malononitrile and indole in presence of Envirocat EPZ-G as a heterogeneous environmental friendly catalyst. This is an environmentally benign method and reusability of the catalyst is beneficial over the others.

Keywords: Oxindoles, EPZ-G and Enviro Catalyst.

(C) RASĀYAN. All rights reserved

\section{INTRODUCTION}

The multicomponent protocol has great applicability as an environmentally benign synthesis. It has minimization of steps, atom economy, high yield, minimization of waste, cost-effective natural availability, high thermal stability and reusability ${ }^{1-9}$ In recent years research has started great attention towards the development of multicomponent organic synthesis by using inorganic material ${ }^{10}$. EPZ-G is one of the versatile inorganic materials that act as an enviro catalyst with heterogeneous and acidic properties owing to the properties EPZG as Lewis acid ${ }^{11}$ reported in the different transformations such as synthesis of nitro olefins $^{12}$, silylation of alcohols $^{13}$, methoxylation of alcohols $^{14}$, aldoximes to nitriles ${ }^{15}$, and Tosylhydrazonea ${ }^{16}$.In this protocol, we have reported a method of 3,3-Disubstituted Oxindoles synthesis using EPZG catalyst as an environmentally benign protocol.

It involves Knoevenagel condensation followed by Michael addition. Many reports have shown that Knoevenagel condensation of aldehyde or ketone with malononitrile was catalyzed by the base, but recently $\mathrm{ZnCl} 2{ }^{17}$, Bismuth Nitrate ${ }^{18}$ and Maxican bentonitrile ${ }^{19} \mathrm{EPZ}^{-\mathrm{G}^{20}}$ were successfully used as an acid catalyst. This prompted as to develop a new strategy of synthesis of 3,3-Disubstituted Oxindoles by Knoevenagel condensation followed by Michael addition.

3,3-Disubstituted Oxindoles is biologically active compound ${ }^{21-25}$ present in many natural products ${ }^{26-29}$ Most of the biologically active compounds ${ }^{30-31}$ have based on indole skeleton.3,3-Disubstituted Oxindoles carry quaternary carbon atom and multiple functional groups that are strong intermediate which helps during the preparation of biologically active compounds ${ }^{32}$ such as anti-HIV ${ }^{33}$, anti-tumor ${ }^{34-37}$, anti-malarial ${ }^{38}$, antimicrobial $^{39}$, anti-tubercular ${ }^{40-41}$, and antimalarial. ${ }^{42}$ To capture these opportunities chemists play a great role in developing of green synthesis of 3,3-Disubstituted Oxindoles. ${ }^{43-48}$

\section{EXPERIMENTAL}

Various substituted isatin and Isatin derivatives (Sigma-Aldrich), malononitrile ((Sigma-Aldrich), indole and its derivatives (Himedia) were uses as received without purification. IR spectra were recorded on FTIR -7600 Lambda Scientific Spectrometer. NMR spectra were recorded on a Bruker AC $400 \mathrm{MHz}$ spectrometer in DMSO $\mathrm{D}_{6}$ using tetramethylsilane as an internal standard material.

\section{General Procedure}

In a $25 \mathrm{ml}$ round, bottom flask mixture of isatin $(1 \mathrm{mmol})$, malononitrile $(1 \mathrm{mmol})$, indole $(1 \mathrm{mmol})$ and $30 \mathrm{mg}$ EPZG catalyst was refluxed in $5 \mathrm{~mL}$ water: ethanol (v/v 70:30) solvent system at about $80^{\circ} \mathrm{C}$ for the desired

Rasayan J. Chem., 13(3), 1735-1743(2020)

http://dx.doi.org/10.31788/ RJC.2020.1335759

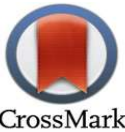


time specified in Table-4. The completion of the reaction was observed by TLC. Upon completion of the reaction separation of the product was carried out by using ethyl acetate. Further purification was carried out by column chromatography using hexane-ethyl acetate $(8: 2) \mathrm{v} / \mathrm{v}$ mixture. All the products were purified by the same technique and were found to be correct. Further structures of the product were confirmed by ${ }^{1} \mathrm{H}$ NMR, ${ }^{13} \mathrm{C}$ NMR and IR.

\section{Spectroscopic Data of 3,3-Disubstituted Oxindoles(Table-1)}

(1a): 2-[3-(1H-indol-3yl)—5-methyl-2-oxoindiln-3yl)]malononitrile

Color: Brown., $T_{\mathrm{mp}}: 220{ }^{\circ} \mathrm{C}, \mathrm{IR}(\mathrm{KBr}) \mathrm{cm}^{-1}: 3360,2923,2258,1743,1480,1287,1774,825 \& 746,{ }^{1} \mathrm{H}$ NMR( $\left.400 \mathrm{MHz}, \mathrm{DMSO}-\mathrm{d}_{6}\right): \delta$, ppm : $2.34(\mathrm{~m}, 3 \mathrm{H}), 5.19(\mathrm{~S}, 1 \mathrm{H},-\mathrm{CH}), 6.91-7.49 \mathrm{ppm}(\mathrm{m}, 8 \mathrm{H} \mathrm{Ar}-\mathrm{H}), 9.94$ (bs, $1 \mathrm{H},-\mathrm{NH}), 10.14$ (bs, $1 \mathrm{H},-\mathrm{N}-\mathrm{H}),{ }^{13} \mathrm{C}$ NMR $\left(100 \mathrm{MHz}, \mathrm{DMSO}-1_{6}\right): \delta$, ppm: 14.08, 22.58, 29.86, 54.08, $110.63,111.40,111.69,111.90,112.11,115.39,120.15,120.19,122.45,124.38,125.32,127.67,135.39$, $137.34,155.92,174.85$.

(1b): 2-[3-(1H-indol-3yl)-2-oxoindiln-3yl)]malononitrile

Color: Brown., $T_{\mathrm{mp}}: 222^{\circ} \mathrm{C}, \mathrm{IR}(\mathrm{KBr}) \mathrm{cm}^{-1}: 3430,3369,2897,2249,1717,1603,1462,1314,1209,1096,1008$, $886 \& 755 .{ }^{1} \mathrm{H}$ NMR $\left(400 \mathrm{MHz}, \mathrm{DMSO}-\mathrm{d}_{6}\right): \delta, \mathrm{ppm}: 5.20(\mathrm{~S}, 1 \mathrm{H},-\mathrm{CH}), 7.0-7.67(\mathrm{~m}, 9 \mathrm{H} \mathrm{Ar}-\mathrm{H}), 9.88$ (bs, $1 \mathrm{H},-\mathrm{NH}), 10.25$ (bs, $1 \mathrm{H},-\mathrm{N}-\mathrm{H}) .{ }^{13} \mathrm{C}$ NMR $\left(100 \mathrm{MHz}, \mathrm{DMSO}-\mathrm{d}_{6}\right): \delta, \mathrm{ppm}: 25.88,53.78,107.87$, $110.63,111.24,111.40,111.84,112.15,114.86,119.07,120.20,121.20,121.61,122.54,123.02,123.16$, $124.36,125.19,125.42,126.56,126.68,130.60,137.32,137.80,142.14,175.02$.

(1c): 2-[3-(1H-indol-3yl)—5-methoxy-2-oxoindiln-3yl)] malononitrile

Color: Brown., $T_{\mathrm{mp}}: 189^{\circ} \mathrm{C}, \mathrm{IR}(\mathrm{KBr}) \mathrm{cm}^{-1}: 3413,3264,2942,2270,1717,1492,1330,1208,875,743,{ }^{1} \mathrm{H}$ $\operatorname{NMR}\left(400 \mathrm{MHz}, \mathrm{DMSO}-\mathrm{d}_{6}\right): \delta \mathrm{ppm}: 3.76\left(\mathrm{~S}, 3 \mathrm{H},-\mathrm{OCH}_{3}\right), 5.18 \mathrm{ppm}(\mathrm{S}, 1 \mathrm{H},-\mathrm{CH}), 6.89-7.52(\mathrm{~m}, 8 \mathrm{H}$ Ar-H), 9.86 (bs, $1 \mathrm{H},-\mathrm{NH}), \delta=10.07$ (bs, $1 \mathrm{H},-\mathrm{N}-\mathrm{H}),{ }^{13} \mathrm{C}$ NMR $\left(100 \mathrm{MHz}, \mathrm{DMSO}-\mathrm{d}_{6}\right): \delta, \mathrm{ppm}: 14.14$, $23.01,31.51,55.79,111.02,111.82,113.58,114.22,117.19,118.43,120.04,121.10,123.17,124.72$, $126.08,134.24,136.09,159.08$.

(1d): 2-[3-(1H-indol-3yl)—5,7-dimethyl-2-oxoindiln-3yl)]malononitrile

Color: Brown. $T_{\mathrm{mp}}: 210^{\circ} \mathrm{C}, \mathrm{IR}(\mathrm{KBr}) \mathrm{cm}^{-1}: 3378,3264,2914,2233,1717,1630,1462,1340,1165,1025$, 921, \& 746, ${ }^{1} \mathrm{H}$ NMR( $\left.400 \mathrm{MHz}, \mathrm{DMSO}-\mathrm{d}_{6}\right): \delta, \mathrm{ppm}: 2.03-2.57$ (m, 6H, 2-CH $), 5.18(\mathrm{~S}, 1 \mathrm{H},-\mathrm{CH})$, 6.84-7.67 (m, 7H Ar-H), 9.43 ppm (bs, 1H, -NH), 9.72 (bs, 1H, N-H), ${ }^{13} \mathrm{C}$ NMR (100 MHz, DMSO $\left.\mathrm{d}_{6}\right): \delta, \mathrm{ppm}=29.70,39.32,55.20,110.99,113.55,114.43,115.13,119.18,119.98,120.06,121.88$, $123.50,124.03,127.05,129.59,133.46,136.19,136.70,158.86,168.81$.

(1e): 2-[3-(1H-indol-3yl)—5-fluoro -2-oxoindiln-3yl)] malononitrile Color: Brown, $T_{\mathrm{mp}}: 240{ }^{\circ} \mathrm{C}$,IR $(\mathrm{KBr}) \mathrm{cm}^{-1}: 3395,3351,1734,1621,1489,1271,1218,816 \& 746,{ }^{1} \mathrm{H}$ NMR (400 MHz, DMSO - $\left.\mathrm{d}_{6}\right): \delta$, ppm : $5.21(\mathrm{~S}, 1 \mathrm{H},-\mathrm{CH}), 6.99-7.55(\mathrm{~m}, 8 \mathrm{H} \mathrm{Ar}-\mathrm{H}), 9.87$ (bs, $\left.-\mathrm{NH}\right), \delta=$ 10.34 (bs, $1 \mathrm{H}, \mathrm{N}-\mathrm{H}){ }^{13} \mathrm{C}$ NMR $\left(100 \mathrm{MHz}, \mathrm{DMSO}-\mathrm{d}_{6}\right): \delta$,ppm : 29.73, 54.12, 107.37, 110.41, 111.13, $112.00,112.08,112.23,113.05,113.31,117.14,117.37,120.06,120.38,122.71,124.20,125.22,127.80$, $127.88,137.34,138.22,138.24,157.72,160.13,174.89$

(1f): 2-[3-(1H-indol-3yl)-5-Choloro -2-oxoindiln-3yl)] malononitrile Color: Brown, $T_{\mathrm{mp}}: 186^{\circ} \mathrm{C}$,IR $(\mathrm{KBr}) \mathrm{cm}^{-1}: 3386,2914,2249,1717,1630,1462,1296,1244,1165,1034$, $825 \& 728,{ }^{1} \mathrm{H}$ NMR $\left(400 \mathrm{MHz}, \mathrm{DMSO}-\mathrm{d}_{6}\right): \delta, \mathrm{ppm}: 5.19(\mathrm{~S}, 1 \mathrm{H},-\mathrm{CH}), \delta=6.89-7.71(\mathrm{~m}, 8 \mathrm{H} \mathrm{Ar}-\mathrm{H}), \delta=$ 8.42-8.45 (bs, $2 \mathrm{H},-2 \mathrm{NH}),{ }^{13} \mathrm{C}$ NMR (100 MHz, DMSO - $\left.\mathrm{d}_{6}\right): \delta$,ppm : 29.88, 53.96, 107.32, 110.36, 111.04, $112.24,112.35,120.11,120.84,122.80,124.15,125.31,125.43,128.05,128.33,130.76,137.34,140.81$, 174.61

(1g): 2-[3-(1H-indol-3yl)—5- Bromo -2-oxoindiln-3yl)] malononitrile

Color: Brown, $T_{\mathrm{mp}}: 190^{\circ} \mathrm{C}, \mathrm{IR}(\mathrm{KBr}) \mathrm{cm}^{-1}: 3386,3264,2932,2242,1717,1612,1498,1323,1183, \& 850,{ }^{1} \mathrm{H}$ NMR (400 MHz, DMSO - $\left.\mathrm{d}_{6}\right): \delta$,ppm : $5.19(\mathrm{~S}, 1 \mathrm{H},-\mathrm{CH}), 6.89-7.86(\mathrm{~m}, 8 \mathrm{H} \mathrm{Ar}-\mathrm{H}), 8.41(\mathrm{bs}, 2 \mathrm{H},-2 \mathrm{NH}),{ }^{13} \mathrm{C}$ 
NMR (100 MHz, DMSO - $\left.\mathrm{d}_{6}\right): \delta, p p m ~: ~ 29.69,53.92,107.34,110.35,111.02,112.24,112.80,120.17$, $120.48,122.79,124.15,125.35,128.11,128.42,133.63,137.38,141.38,174.39$.

(1h): 2-[3-(2-methyl-1H-indol-3yl) -5- methoxy-2-oxoindiln-3yl)] malononitrile

Color: Brown. $T_{\mathrm{mp}}: 212{ }^{\circ} \mathrm{C}, \mathrm{IR}(\mathrm{KBr}) \mathrm{cm}^{-1}: 3308,2949,2189,1717,1489,1296,1192,999,825 \& 737,{ }^{1} \mathrm{H}$ $\operatorname{NMR}\left(400 \mathrm{MHz}, \mathrm{DMSO}-\mathrm{d}_{6}\right): \delta, \mathrm{ppm}: 2.37\left(\mathrm{~S}, 3 \mathrm{H},-\mathrm{CH}_{3}\right), 3.14\left(\mathrm{~S}, 3 \mathrm{H},-\mathrm{OCH}_{3}\right), 5.42(\mathrm{~S}, 1 \mathrm{H},-\mathrm{CH}), 6.78-$ $7.03(\mathrm{~m}, 7 \mathrm{H}, \mathrm{Ar}-\mathrm{H}), 9.60(\mathrm{bs}, 1 \mathrm{H},-\mathrm{NH}), 9.94 \mathrm{ppm}(\mathrm{bs}, 1 \mathrm{H},-\mathrm{N}-\mathrm{H}){ }^{13} \mathrm{C} \mathrm{NMR}\left(100 \mathrm{MHz}, \mathrm{DMSO}-\mathrm{d}_{6}\right): \delta, \mathrm{ppm}$ : $14.31,30.44,54.54,55.75,110.61,111.05,111.61,111.90,112.05,115.63,118.98,119.61,120.99$, $126.57,128.89,134.38,135.12,135.62,155.85,175.51$

(1i): 2-[3-(2-methyl-1H-indol-3yl)—5-bromo-2-oxoindiln-3yl)] malononitrile Color: Brown, $T_{\mathrm{mp}}: 186^{\circ} \mathrm{C}, \mathrm{IR}(\mathrm{KBr}) \mathrm{cm}^{-1}: 3386,2923,2249,1708,1603,1480,1287,1156,1113,825$ \& $746,{ }^{1} \mathrm{H}$ NMR $\left(400 \mathrm{MHz}, \mathrm{DMSO}-\mathrm{d}_{6}\right): \delta$, ppm : $1.60\left(\mathrm{~s}, 3 \mathrm{H},-\mathrm{CH}_{3}\right) 5.42(\mathrm{~s}, 1 \mathrm{H},-\mathrm{CH}), 6.91-7.83(\mathrm{~m}, 7 \mathrm{H} \mathrm{Ar}-$ H), 7.97 (bs, $1 \mathrm{H},-\mathrm{NH}), 8.12$ (bs, $1 \mathrm{H},-\mathrm{N}-\mathrm{H}),{ }^{13} \mathrm{C}$ NMR $\left(100 \mathrm{MHz}, \mathrm{DMSO}-\mathrm{d}_{6}\right): \delta, \mathrm{ppm}: 30.11,52.00,99.15$, $111.03,112.03,112.39,116.42,120.76,122.25,126.54,129.26,134.08,140.04,147.82,171.00$.

(1j): 2-[3-(2-methyl-1H-indol-3yl)-5,7-dimethyl-2-oxoindiln-3yl)] malononitrile

Color: Brown, $T_{\mathrm{mp}}: 228^{\circ} \mathrm{C}$,IR $(\mathrm{KBr}) \mathrm{cm}^{-1}: 3370,3219,2909,2245,1717,1645,14921360,1165,975$, $850 \& 741,{ }^{1} \mathrm{H}$ NMR $\left(400 \mathrm{MHz}, \mathrm{DMSO}-\mathrm{d}_{6}\right): \delta, \mathrm{ppm}: 2.17-2.47\left(\mathrm{~m}, 9 \mathrm{H}, 3-\mathrm{CH}_{3}\right), 5.46(\mathrm{~S}, 1 \mathrm{H},-\mathrm{CH}), 6.79-$ 7.90 (m, 6H, Ar-H), 8.07 (bs, $2 \mathrm{H}-2 \mathrm{NH}){ }^{13} \mathrm{C}$ NMR $\left(100 \mathrm{MHz}, \mathrm{DMSO}-\mathrm{d}_{6}\right): \delta, \mathrm{ppm}: 38.02,55.78,111.45$, $112.19,114.51,115.38,115.88,116.08,118.76,119.54,121.16,22.28,123.98,127.04,129.16,130.12$, $131.10,132.04,133.32,133.94,134.74,135.15,137.84,144.58,149.55,163.15$ ppm.

\section{(1k): 2-[3-(2-methyl-1H-indol-3yl)- 2-oxoindiln-3yl)]malononitrile}

Color: Brown, $T_{\mathrm{mp}}: 180^{\circ} \mathrm{C}, \mathrm{IR}(\mathrm{KBr}) \mathrm{cm}^{-1}: 3339,3260,2927,2219,1717,1630,1490,1289,1172,900 \&$ $749,{ }^{1} \mathrm{H}$ NMR $\left(400 \mathrm{MHz}, \mathrm{DMSO}-\mathrm{d}_{6}\right): \delta$,ppm : 2.28-2.79 (m, 3H, $\left.-\mathrm{CH}_{3}\right), 5.96(\mathrm{~s}, 1 \mathrm{H},-\mathrm{CH}), 6.83-7.73(\mathrm{~m}$, $8 \mathrm{H}, \mathrm{Ar}-\mathrm{H}), 10.90$ (bs, $2 \mathrm{H}, 2 \mathrm{~N}-\mathrm{H}),{ }^{13} \mathrm{C}$ NMR $\left(100 \mathrm{MHz}, \mathrm{DMSO}-\mathrm{d}_{6}\right): \delta, \mathrm{ppm}=28.12,57.44,111.45,112.44$, $114.92,115.33$, 116.91, 117.42, 118.76, 119.20, 121.12, 112.53, 123.98, 124.06, 126.72, 127.04, 130.07, $134.04,137.84,144.58,149.55,163.34$.

(11): 2-[3-(2-methyl-1H-indol-3yl) -5-iodo-2-oxoindiln-3yl)] malononitrile

Color: Brown, $T_{\mathrm{mp}}: 216^{\circ} \mathrm{C}, \mathrm{IR}(\mathrm{KBr}) \mathrm{cm}^{-1}: 3374,3210,2930,2233,1716,1608,1466,1342,1204,766$ \& $710,{ }^{1} \mathrm{H}$ NMR (400 MHz, DMSO - $\left.\mathrm{d}_{6}\right): \delta$, ppm : $2.32\left(\mathrm{~s}, 3 \mathrm{H},-\mathrm{CH}_{3}\right), 5.44(\mathrm{~s}, 1 \mathrm{H},-\mathrm{CH}), 6.84-8.08(\mathrm{~m}, 8 \mathrm{H} \mathrm{Ar}-$ $\mathrm{H}),{ }^{13} \mathrm{C}$ NMR $\left(100 \mathrm{MHz}, \mathrm{DMSO}-\mathrm{d}_{6}\right): \delta$,ppm : 30.23, 52.09, 111.04, 111.93, 111.09, 120.75, 120.93 , $122.25,126.53,131.19,137.43,138.08,151.96,175.07$.

(1m): 2-[3-(2-methyl-1H-indol-3yl)-5-methyl-2-oxoindiln-3yl)] malononitrile

Color: Brown, $T_{\mathrm{mp}}: 260^{\circ} \mathrm{C}, \mathrm{IR}(\mathrm{KBr}) \mathrm{cm}^{-1}: 3344,3298,2953,2284,1717,1631,1488,1217,1169,763$ \& $629,{ }_{1}^{1} \mathrm{H}$ NMR $\left(400 \mathrm{MHz}, \mathrm{DMSO}-\mathrm{d}_{6}\right): \delta, \mathrm{ppm}: 2.35\left(\mathrm{~m}, 6 \mathrm{H}, 2-\mathrm{CH}_{3}\right), 5.44(\mathrm{~s}, 1 \mathrm{H},-\mathrm{CH}), 6.76-7.81(\mathrm{~m}, 8 \mathrm{H}$, $\mathrm{Ar}-\mathrm{H}), 9.67$ (bs, 1H, -NH), 10.31 (bs, $1 \mathrm{H}, \mathrm{N}-\mathrm{H}),{ }^{13} \mathrm{C}$ NMR (100 MHz, DMSO - $\left.\mathrm{d}_{6}\right): \delta$,ppm : 29.34, 40.52, $54.82,54.90,111.92,112.17,113.14,114.89,118.22,118.92,119.68,120.33,122.41,123.07,125.03$, $127.75,133.12,134.76,136.88,159.04$.

\section{RESULTS AND DISCUSSION}

We have developed the synthesis of 3,3-Disubstituted Oxindoles via one-pot multi-component condensation of indole, malononitrile and isatin by using EPZ-G as a catalyst and in presence of mixed solvent system (Water: Ethanol70: $30 \mathrm{v} / \mathrm{v}$ ) under reflux condition This is an expeditious procedure gives yield the fine product (Scheme-1). The results were obtained summarized in Table-1.

As the reaction was carried out with the different substituents in indole and isatin revealed delicate electronic effect, electron-donating groups deactivates the isatin as well as indole nucleus like methyl and methoxy group (entries 3 and 4, Table-1). Due to this prolonged reaction time and offered corresponding less yield of the product. Reacting nucleus bearing electron-withdrawing group such as $\mathrm{Br}, \mathrm{F}$ (entries 5 and 
RASĀYAN J. Chem.

Vol. 13 | No. 3 |1735-1743| July - September | 2020

6, Table-1) reacts much faster and offered an excellent yield of the product. Perhaps low yield of product was due to the steric hindrance offered in 2-substituted indole (entries 8 to 13, Table-1)

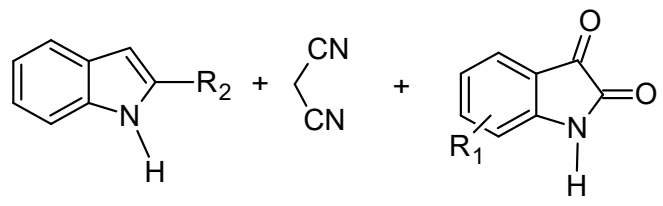

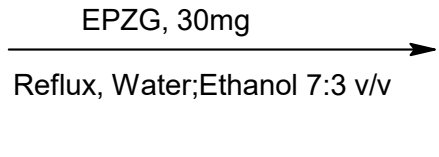<smiles>[R1]Cc1ccccc1C1(c2c([R2])[nH]c3ccccc23)C(=O)Nc2ccccc21</smiles>

Scheme-1

Table-1: One-pot Multicomponent Synthesis of 3,3-Disubstituted Oxindoles

Entry


RASĀYAN J. Chem.

Vol. 13 | No. 3 |1735-1743| July - September | 2020

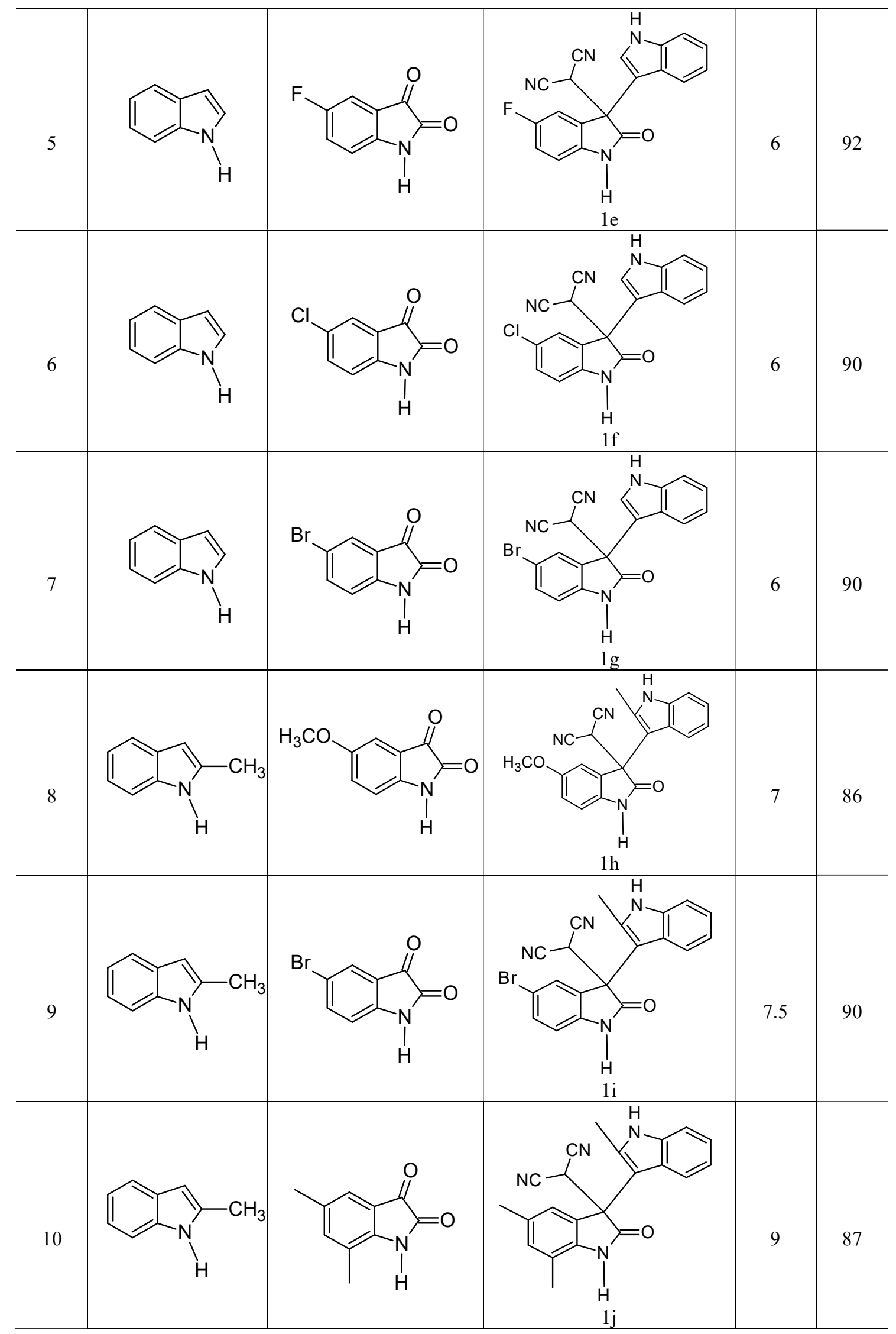


RASĀYAN J. Chem.

Vol. 13 | No. 3 |1735-1743| July - September | 2020

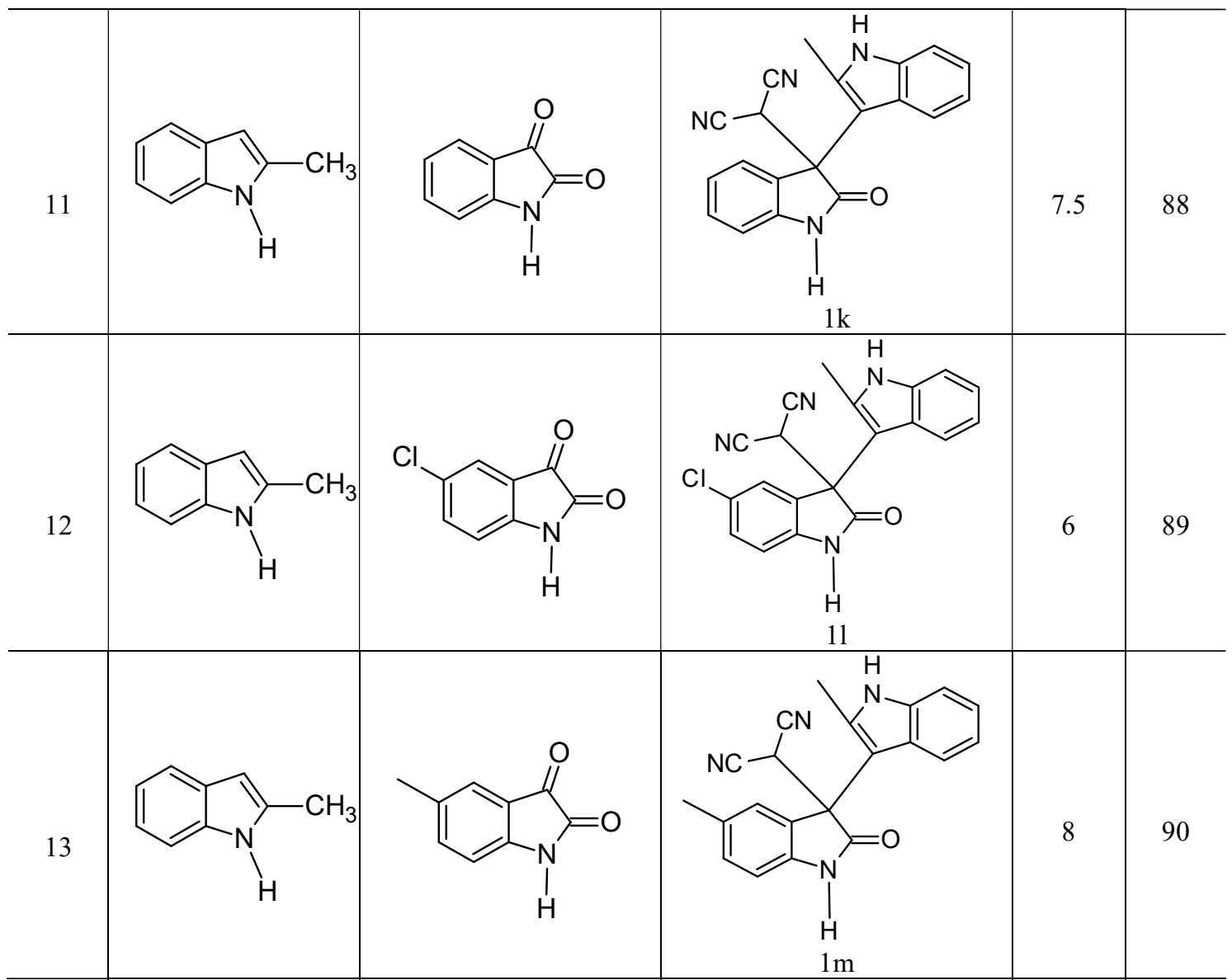

Reaction Condition: Indole (1mmol), isatin $(1 \mathrm{mmol})$ and malononitrile $(1 \mathrm{mmol})$ reflux in $5 \mathrm{~mL}$ water: ethanol $(\mathrm{v} / \mathrm{v}$ 70:30) solvent system, EPZ-G 30mg.

The results were obtained for 3,3-Disubstituted Oxindoles was summarized in Table-1, it was clear that the amount of catalyst increases yield of the product also increases with decreasing in reaction time, $30 \mathrm{mg}$ of the catalyst is sufficient for the reaction to obtained maximum yield (Entry 5, Table-2). Whenever amount of catalyst increases i.e. $35 \mathrm{mg}$ and $40 \mathrm{mg}$ no significant change in the reaction time and yield (Entry 6 and 7, Table-2). While amount of catalyst decreases then it effects the reaction time and yield. (Entry 1, 2, 3 and 4 , Table-2).

Table-2: Screening of Catalyst

\begin{tabular}{c|c|c|c|c}
\hline S. No. & Catalyst & $\begin{array}{c}\text { Mol } \\
\%\end{array}$ & $\begin{array}{c}\text { Time } \\
(\mathrm{h})\end{array}$ & $\begin{array}{c}\text { Yield } \\
(\%)\end{array}$ \\
\hline 1 & EPZ-G & 10 & 12 & 82 \\
\hline 2 & EPZ-G & 15 & 10 & 84 \\
\hline 3 & EPZ-G & 20 & 09 & 84 \\
\hline 4 & EPZ-G & 25 & 08 & 88 \\
\hline 5 & EPZ-G & 30 & 6 & 92 \\
\hline 6 & EPZ-G & 35 & 6 & 90 \\
\hline 7 & EPZ-G & 40 & 6 & 90 \\
\hline
\end{tabular}

Reaction Condition: Indole $(1 \mathrm{mmol})$, isatin $(1 \mathrm{mmol})$ and malononitrile $(1 \mathrm{mmol})$ reflux in $5 \mathrm{~mL}$ water: ethanol $(\mathrm{v} / \mathrm{v}$ 70:30) solvent system, EPZ-G 30mg.

Further extension of the study towards the mixed solvent system and it has been proved that mixed solvent system was most powerful than the single solvent, the same reaction was carried out with different 
RASĀYAN J. Chem.

Vol. 13 | No. 3 |1735-1743| July - September | 2020

proportion of ethanol and water as a solvent system then wonderful results were obtained for the same solvent system (Table-3).

To develop an aqueous solvent system model reaction was carried out in a universal solvent, i.e. water (entry 1, Table-3). But the result achieved was very poor, so the reaction was carried out in pure ethanol medium then also yield and reaction time was not good. After that we have tried for the mixed solvent system $^{5}$ and compared its result (Table-3), and it was found that mixed solvent system plays an important role during the formation of product and mixed solvent system (entry 4, Table-3) gives an excellent yield of the product within comparable reaction time. The product was separated by ethyl acetate to remove the catalyst.

Table-3: Use of Different Solvent Systems

\begin{tabular}{c|c|c|c|c}
\hline S. No. & Solvent & Solvent System $(\%)$ & Time $(\mathrm{h})$ & Yield (\%) \\
\hline 1 & Water & 100 & 10 & 54 \\
\hline 2 & Water: ethanol & $90: 10$ & 8 & 61 \\
\hline 3 & Water: ethanol & $80: 20$ & 8 & 82 \\
\hline 4 & Water: ethanol & $70: 30$ & 6 & 92 \\
\hline 5 & Water: ethanol & $60: 40$ & 7 & 87 \\
\hline 6 & Water: ethanol & $50: 50$ & 7 & 87 \\
\hline 7 & Water: ethanol & $40: 60$ & 8 & 83 \\
\hline 8 & Water: ethanol & $30: 70$ & 9 & 82 \\
\hline 9 & Water: ethanol & $20: 80$ & 10 & 82 \\
\hline 10 & Water: ethanol & $10: 90$ & 10 & 81 \\
\hline 11 & Ethanol & 100 & 11 & 80 \\
\hline
\end{tabular}

Reaction Condition: Indole $(1 \mathrm{mmol})$, isatin $(1 \mathrm{mmol})$ and malononitrile $(1 \mathrm{mmol})$ reflux in $5 \mathrm{mLwater}$ : ethanol $(\mathrm{v} / \mathrm{v}$ 70:30) solvent system using30mg of EPZ-G catalyst.

\section{Reusability of Catalyst}

After the recovery catalyst was washed with ethyl acetate then with water, dried well in the oven, and reused for further reaction. Reusability studied and result achieved summarized in table 4 (entry 1-6, Table-3). From the results, it was clear that excellent reusability of the catalyst after five successive transformations, after that yield of the product decreases due to leaching out of the catalyst.

Table-4: The Reusability of EPZG.

\begin{tabular}{c|c|c}
\hline S. No. & Time $(\mathrm{h})$ & Yield (\%) \\
\hline 1 & 6 & 92 \\
\hline 2 & 6 & 90 \\
\hline 3 & 6 & 88 \\
\hline 4 & 7 & 87 \\
\hline 5 & 7 & 85 \\
\hline 6 & 9 & 72
\end{tabular}

Reaction Condition: Indole (1 $\mathrm{mmol})$, isatin (1mmol) and malononitrile $(1 \mathrm{mmol})$ reflux in $5 \mathrm{~mL}$ water: ethanol $(\mathrm{v} / \mathrm{v}$ 70:30) solvent system using 30mg of EPZ-G catalyst.

\section{CONCLUSION}

We have developed a one-pot protocol for the 3,3-Disubstituted Oxindoles under a mixed solvent system (water: ethanol,3:7v/v) catalyzed by $30 \mathrm{mg}$ of EPZG. In this context, EPZG as a Lewis acid signifies remarkable performance due to electrophilic nature. The demand for this catalyst not only for maintainable yield but also to replace the use of toxic catalysts or solvents. Moreover, eco-friendly, simple workup procedure, reusability of the catalyst makes this protocol acceptable methodology.

\section{REFERENCES}

1. A. Strecker, Justus Liebigs Annalen Der Chemie,75, 27(1850), DOI:10.1002/9780470638859

2. I. Ugi, Advanced Synthesis and Catalysis, 339, 499(1997).

3. N. K.Terret, M. Gardener, D. W. Gordon, R. J. Kobylecki, J., Steele, Tetrahedron, 51, 8135(1995), DOI: 10.1016/S0040-4039(97)00986-6 
RASĀYAN J. Chem.

Vol. 13 | No. 3 |1735-1743| July - September | 2020

4. A. Domling, I. Ugi, Angewanted Chemie International Edition, 39, 3168 (2000), DOI:10.1002/15213773(20000915)39:183.0.

5. A. Domling, Chemical Reviews, 106, 17(2006), DOI:10.1021/cr0505728

6. R. W. Armstrong, A. P. Combs, P. A. Tempest, S. D. Brown, T. A. Keating, Accounts of Chemical Research, 29, 132 (1996).

7. F. L. Muller, T. Constantieux, J. Rodriguez, Journal of The American Chemical Society,127, 171(2005), DOI: $10.1021 / \mathrm{ja} 0570032$

8. B., Willy, T. J. Muller, European Journal of Organic Chemistry, 2008(24), 4157(2008), DOI: 10.1002/ejoc.200800444

9. M, Adib, E. Sheikhi, A. kavoosi, H. R. Bijanzadeh, Tetrahedron, 66, 9263(2010), DOI: $10.1002 / \mathrm{hlca} .201300384$

10. J. H. Clark, A. P. Kybett, and D. J. Macaquarrie, Supported Reagents, VCH, New York 1992, DOI: 10.1002/ange.19941060433

11. B.P. Bandgar, M. B. Zirange, P. P. Wadagaonkar, Synlett.,2, 149 (1996), DOI:10.1055/s-1996-5347

12. B. P. Bandgar, P. P. Wadagaonkar, Synthetic Communications, 27, 2069(1997), DOI: $10.1002 /$ jecs. 200700068

13. B. P. Bandgar, C. T. Hajare, P. P. Wadagaonkar, Journal of Chemical Research Synopses, 1, 90 (1995), DOI: $10.1002 /$ jecs. 200000170

14. B. P. Bandgar, S. R. Jagtap, S. B.Ghodeshwar, P. P. Wadagaonkar, Synthetic Communications, 25, 2993 (1995), DOI: $10.1081 /$ SCC-120005936

15. R. Ballini, G. Boscia, R. Maggi, G. Sartori, Synlett, 8,795(1997).

16. T. Green, W. Wuts., PGM Protective Group in Organic Synthesis, $2^{\text {nd }}$ edn. Wiley, New York, 178, (1991), DOI:10.1021/jm990518h

17. F.A.J. Meskens, Synthesis, 5,501(1981).

18. E. Taylor, C. Chiang, Synthesis, 7,467 (1977), DOI: 10.1021/j100540a008

19. B. P. Bandgar, S. M. Zirange, P. P. Wadgaonkar, Synthetic Communications, 27(7), 1153(1997), DOI: $10.1080 / 00397919708003351$

20. C. Marti, M. Carreria, European Journal of Organic Chemistry, 2209(2003), DOI: $10.1002 /$ ejoc. 200300050

21. S. Hibino, T. Choshi, Natural Product Reports, 18, 66(2001).

22. H Lin, S. J. Danishefsky, Angewanted Chemie International Edition, 42, 36(2003), DOI: 10.1002 /anie. 201201736

23. M. Petterson, D. KnueppelS. F., Martin, Organic Letters, 9, 4623(2007), DOI:10.1021/o1702132

24. D. Ravelli, D. Dondi, M. Fagnoni, A. Albini, Chemical Society Reviews,38, 1999(2009).

25. H. Deppermann, A. H. Thomanek, G. P. Prenzel, W. J. Maison, Journal of Organic Chemistry, 75, 5994 (2010), DOI:10.1021/jo101401

26. Z. Y. Cao, Y. H. Wang, X. P. Zeng, J. Zhou, Tetrahedron Letter, 55, 2571(2014), DOI: $10.1002 /$ adsc. 201700361

27. S. Wang, S. Yu, W. Sun, S. K. Shangary, P. D. Sun, D. McEachern, Y. Zhao, Patent, U. S., 2011052 (2011).

28. G. Zeni, R. C. Larock, Chemical Reviews, 106, 4644 (2006), DOI:10.1021/cr020085h

29. C. V. Galliford, K. A. Scheidt, Angewanted Chemie International Edition,46, 8748(2007), DOI: 10.1002/anie.200701342

30. S. Ma, X. Han, S. Krishna, S. C. Virgil, B. M. Stoltz, Angewanted Chemie International Edition,48, 8037(2009), DOI: 10.1021/cr020039h

31. A. Fensome, W. R. Adams, A. L. Adams, T. J. Berrodin, J. Cohen, C. Huselton, A.Illenbeger, J. C. Kern, V. A. Hudak, M. A. Marella, E. G. Melenski, C. C McComas,C. A. Mugford, O. D. Slayden, M. Yudt, Z. Zhang, Y. Zhu, R. C. Winneker, Journal of Medicinal Chemistry, 51, 1861(2008).

32. B.K. Paul, D. Ray, N. Guchhait, Physical Chemistry Chemical Physics, 15, 1275 (2013)

33. D. Havrylyuk, B. Zimenkovsky, O. Vasylenko, A. Gzella, R. Lesyk, Journal Of Medicinal Chemistry,55, 8630(2012), DOI:10.1021/jm300789g 
RASĀYAN J. Chem.

Vol. 13 | No. 3 |1735-1743| July - September | 2020

34. C. Liang, J. Xia, D. Lei, X. Li, Q. Yao, J. Gao, European Journal of Medicinal Chemistry,74, 742, 2014.

35. S. B. Kumar, M. Ravindra, G. Kishore, V. J. Rao, P.Yogeeswari,D. Sriram, Medicinal Chemistry Research, 23,1934 (2014).

36. K. Han, Y. Zhou, F. Liu, Q. Guo, P. Wang, Y. Yang, B. Song, W. Liu, Q. Yao, P. Yu. Teng, Bioorganic and Medicinal Chemistry Letters, 24, 591 (2014), DOI:10.1016/j.bmcl.2013.12.001

37. R. Raj, P. Singh, J. Gut,P. J. Rosenthal, V. Kumar, Europian Journal of Medicinal Chemistry, 62, 590 (2013), DOI:10.1016/j.ejmech.2013.01.032

38. M. Kumar, K. Ramasamy, V. Mani, R. K. Mishra, A. B. Majeed, E. D. Clercq, B.Narasimhan, Arabian Journal of Chemistry, 7, 396 (2014).

39. K. Kumar, S. Carrere-Kremer, L. Kremer, Y. Gueerardel, C. Biot, V. Kumar, Organometallics, 32, 5713 (2013).

40. P. Mondal, S. Jana, A. Balaji, R. Ramakrishna, L. K. Kanthal, Journal of Young Pharmacists, 4, 38 (2012).

41. G. Kiran, T. Maneshwar, Y. Rajeshwar, M. Sarangapani, Journal of Chemistry, 2013, DOI: $10.1155 / 2013 / 192039$

42. A. Millemaggi, J. K. Taylor, European Journal of Organic Chemistry, 4527(2010), DOI: $10.1155 / 2013 / 192039$

43. F. Zhou, Y.- L. Liu, J. Zhou, Advanced Synthesis and Catalysis,352, 1381(2010), DOI: 10.1002/adsc.201000161

44. T. Zhang, L. Cheng, S. Hammed, L. Liu, D. Wang, Y. J. Chen, Chemical Communications, 47, 6644(2011).

45. G. Bencivenni, L.-Y. Wu, A. Mazzanti, B. Giannichi, F.Pesciaioli, M. P. Song, G.Bartoli, P. Melchiorre, Angewanted Chemie International Edition, 48, 7200(2009), DOI: 10.1002/anie.200903192

46. L. L. Wang, L. Peng, J.-F. Bai, L. N. Jia, X. Y. Luo, Xu. X. Y. Huang, L. X. Wang, Chemical Communications, 47, 5593(2011), DOI:10.1002/asia.201201244

47. X. Jiang, Y. Sun, J. Cao, M. kai, N. He, X. Zhang, Y. Wang, R. Wang, Advanced Synthesis And Catalysis,354, 917(2012).

[RJC-5759/2020] 